\title{
Diagnosis of complications associated with acute cholecystitis using computed tomography and diffusion-weighted imaging with background body signal suppression/T2 image fusion
}

\author{
MINORU TOMIZAWA ${ }^{1}$, FUMINOBU SHINOZAKI ${ }^{2}$, SATOMI TANAKA ${ }^{2}$, TAKAFUMI SUNAOSHI ${ }^{2}$, \\ DAISUKE KANO ${ }^{2}$, ERIKO SUGIYAMA ${ }^{2}$, MISAKI SHITE ${ }^{2}$, RYOUTA HAGA ${ }^{2}$, YOSHIYA FUKAMIZU ${ }^{2}$, \\ TOSHIYUKI FUJITA ${ }^{2}$, SATOSHI KAGAYAMA ${ }^{2}$, RUMIKO HASEGAWA ${ }^{3}$, YOSHINORI SHIRAI ${ }^{3}$, \\ YASUFUMI MOTOYOSHI ${ }^{4}$, TAKAO SUGIYAMA ${ }^{5}$, SHIGENORI YAMAMOTO $^{6}$ and NAOKI ISHIGE ${ }^{7}$ \\ Departments of ${ }^{1}$ Gastroenterology, ${ }^{2}$ Radiology, ${ }^{3}$ Surgery, ${ }^{4}$ Neurology, ${ }^{5}$ Rheumatology, ${ }^{6}$ Pediatrics and ${ }^{7}$ Neurosurgery, \\ National Hospital Organization, Shimoshizu Hospital, Yotsukaido, Chiba 284-0003, Japan
}

Received April 2, 2016; Accepted March 17, 2017

DOI: $10.3892 / e t m .2017 .4567$

\begin{abstract}
In a clinical setting, it is important to diagnose complications of acute cholecystitis accurately. Diffusion-weighted whole body imaging with background body signal suppression/T2-weighted image fusion (DWIBS/T2) provides high signal intensity with a strong contrast against surrounding tissues in anatomical settings. In the present study, patients who were being treated for acute cholecystitis and underwent DWIBS/T2 in the National Hospital Organization Shimoshizu Hospital between December 2012 and August 2015 were enrolled. A total of 10 men and 4 women underwent DWIBS/T2. Records, including DWIBS/T2 and computed tomography (CT) imaging, were retrospectively analyzed for patients with acute cholecystitis. CT images revealed thickened gallbladder walls in patients with acute cholecystitis, and high signal intensity was observed in DWIBS/T2 images for the thickened gallbladder wall. Inflammation of the pericholecystic space and the liver resulted in high intensity signals with DWIBS/T2 imaging, whereas CT imaging revealed a low-density area in the cholecystic space. Plain CT scanning identified a low-density area in the liver, which became more obvious with contrast-enhanced CT. DWIBS/T2 imaging showed the inflammation of the liver and pericholesyctic space as an area of high signal intensity. Detectability of inflammation of the pericholecystic space and the liver was the same for DWIBS/T2 and CT, which suggests that DWIBS/T2 has the same sensitivity as CT scanning for the diagnosis of complicated acute cholecystitis. However, the strong contrast shown
\end{abstract}

Correspondence to: Dr Minoru Tomizawa, Department of Gastroenterology, National Hospital Organization, Shimoshizu Hospital, 934-5 Shikawatashi, Yotsukaido, Chiba 284-0003, Japan E-mail: nihminor-cib@umin.ac.jp

Key words: acute cholecystitis, computed tomography, liver abscess, peritonitis by DWIBS/T2 allows for easier evaluation of acute cholecystitis than CT scanning.

\section{Introduction}

Acute cholecystitis is an inflammation of the gallbladder caused by gallstones packed in its neck or the cystic duct (1). Perforations of the gallbladder into the liver or the pericholecystic space may lead to the development of liver abscesses or peritonitis, respectively (2-4). In order to effectively manage acute cholecystitis, an accurate diagnosis must be made before the disease worsens (5). Acute cholecystitis is diagnosed based on a combination of signs indicating local and systemic inflammation (6). Local inflammation presents as right upper quadrant pain. Murphy's sign, which is a pain on taking a deep breath in the right upper quadrant when the examiner's finger is on the location of the gallbladder, is considered to be the most useful indicator for the diagnosis of local inflammation in patients with acute cholecystitis (7). Systemic inflammation is confirmed based on the findings of blood tests, with leukocytosis and elevated levels of C-reactive protein (CRP) indicating systemic inflammation (8). To confirm the diagnosis of cholecystitis, diagnostic imaging is useful; computed tomography (CT) scanning typically reveals thickened walls of the gallbladder, pericholecystic inflammation, and the presence of liver abscesses (9).

Diffusion-weighted imaging (DWI) utilizes the random movement of water molecules to construct images (10). Based on DWI, diffusion-weighted whole body imaging with background body signal suppression (DWIBS) has been developed $(11,12)$. DWIBS images are formed with the suppression of fat signals and the application of heavy diffusion weighting during free breathing (13). DWIBS shows a strong contrast between $r$ tissue with high signal intensity and the surrounding tissues (14). One limitation of DWIBS in some cases is the difficulty of evaluating high signal intensities in anatomical settings $(15,16)$. To overcome this disadvantage, DWIBS images are overlapped with T2-weighted images to create DWIBS T2-weighted image 
Table I. Pulse sequences used in the present study.

\begin{tabular}{|c|c|c|c|}
\hline Variable & T1-weighted image & T2-weighted image & DWI (DWIBS) \\
\hline & GRE & Single-shot SE & EPI SE \\
\hline TR, msec & Shortest & 1,000 & 11,250 \\
\hline TE, msec & First: 2.3 (out of phase), second: 4.6 (in phase) & 90 & 83 \\
\hline Flip angle, ${ }^{\circ}$ & 75 & 90 & 90 \\
\hline NSA & 1 & 1 & 4 \\
\hline Slice thickness, mm & 8 & 8 & 5 \\
\hline Slice gap & 1 & 1 & 0 \\
\hline Fat saturation & No & No & SPAIR \\
\hline Phase encoding direction & Posterior-anterior & Posterior-anterior & Posterior-anterior \\
\hline
\end{tabular}

fusion (DWIBS/T2) $(14,17,18)$. DWIBS/T2 enables the evaluation of tissues with high signal and strong contrast in fusion with T2-weighted images (19).

In the present study, the use of DWIBS/T2 and CT scanning for patients with acute cholecystitis was evaluated.

\section{Materials and methods}

Ethical statement. The Ethics Committee of the National Hospital Organization, Shimoshizu Hospital (Yotsukaidu, Japan) approved the present study. Patient records were anonymous and retrospectively analyzed. Written informed consent was obtained from all the patients prior to DWIBS/T2 and CT, with or without contrast enhancement, being performed.

Patients. Patients who were treated for acute cholecystitis and underwent DWIBS/T2 in the National Hospital Organization, Shimoshizu Hospital between December 2012 and August 2015, and for whom DWIBS/T2 and CT results were available, were enrolled in the present study. Ten men (aged $67.7 \pm 7.6$ years) and 4 women (aged 70.8 \pm 13.2 years) were enrolled in the present study. No patients were treated for acute cholecystitis prior to DWIBS/T2 and CT.

Diagnosis of acute cholecystitis. Diagnosis of acute cholecystitis was based on Tokyo Guideline 13 (6). Patients were suspected of acute cholecystitis when they presented with upper abdominal pain or right upper quadrant abdominal pain accompanied by leukocytosis or elevated CRP levels. Patients were subjected to abdominal ultrasonography or CT scanning, with or without contrast enhancement. Abdominal ultrasonography and CT have previously been demonstrated to detect an enlarged gallbladder, thickened walls, and fluid collection between the liver and gallbladder or pericholecystic space (20). A positive sonographic for Murphy's sign was a reliable test for the diagnosis of acute cholecystitis (21).

Magnetic resonance imaging (MRI) techniques. The MRI studies were performed using a 1.5 Tesla scanner with
Achieva software version 3.2.2 (Philips Healthcare, DA Best, The Netherlands). Patients were placed in a supine headfirst position on an extended table platform that allowed for coverage of the body from the head to the lower legs. The DWIBS/T2 imaging protocol consisted of unenhanced T1-weighted, T2-weighted, DWI, and DWIBS imaging. The MRI pulse sequences are presented in Table I. DWIBS images were acquired axially by a Q-body coil, under free breathing conditions, using an echo-planar imaging single-shot pulse sequence. DWI gradients were applied along the $\mathrm{X}, \mathrm{Y}$, and $\mathrm{Z}$ axes prior to and following a $180^{\circ}$ inversion pre-pulse to obtain fat-saturated isotropic images with DWI sensitivity. The following parameters were used for a single stack: B-value, 0 and $800 \mathrm{~mm}^{2} / \mathrm{sec}$; repetition time, $6,960 \mathrm{msec}$; echo time, $7 \mathrm{msec}$; inversion recovery, $150 \mathrm{msec}$; acquisition matrix, 176x115; reconstruction matrix, 256; right/left field of view, $530 \mathrm{~mm}$; anterior/posterior field of view, $349 \mathrm{~mm}$; feet/head field of view, $226 \mathrm{~mm}$; slice thickness, $6 \mathrm{~mm}$; and size of reconstructed voxels, 2.07x2.08x6 $\mathrm{mm}^{3}$. Fused DWIBS/T2 images were constructed using an Extended MR WorkSpace workstation (Phillips Healthcare). For all patients, five stacks were acquired consecutively to obtain images from the head to the middle of the tibia, with each stack consisting of 45 slices. Overall, the average required imaging time was $13.31 \mathrm{~min}$. To rule out $\mathrm{T} 2$ shine-through effects and to differentiate malignant lesions from non-malignant causes of restricted diffusion, a decreased signal on the apparent diffusion coefficient (ADC) was used with ADC reduction, to determine a 'positive ADC map' (22).

CT scanning. CT scanning was performed using a 16-detector row CT scanner (SOMATOM Emotion 16; Siemens AG, Munich, Germany). Imaging parameters for three-phase contrast-enhanced images were as follows: Tube voltage, $130 \mathrm{kVp}$; gantry rotation speed, 0.6 rotations/sec; maximum allowable tube current, $120 \mathrm{~mA}$. For some patients, contrast medium (100 ml of iopamidol; $3 \mathrm{ml} / \mathrm{sec}$; Konica Minolta, Inc., Tokyo, Japan) was administered intravenously. CT images were acquired prior to and at 30, 70, and $180 \mathrm{sec}$ following injection of contrast medium. 

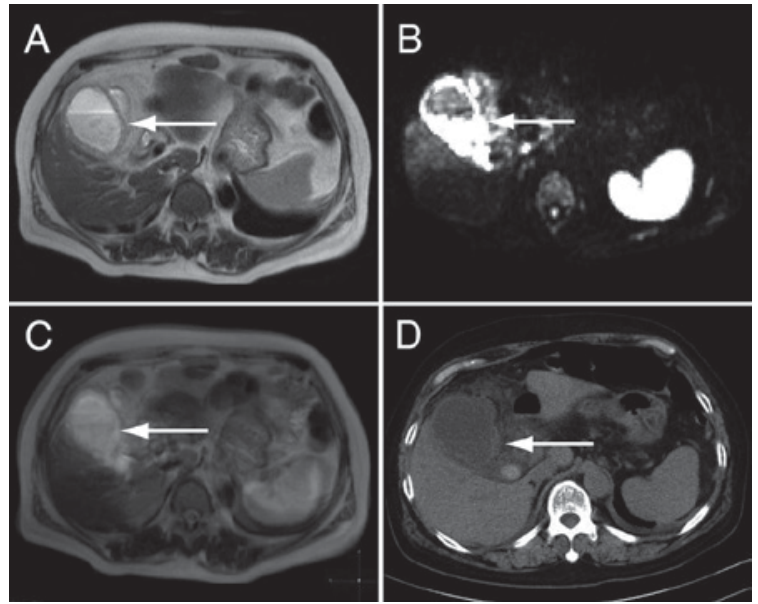

Figure 1. Thickening and high signal intensity in the gallbladder wall of a patient with acute cholecystitis. A 70-year-old woman presented with upper abdominal pain. (A) T2-weighted imaging revealed a thickened gallbladder wall. (B) High signal intensity in the gallbladder wall was observed with DWIBS. (C) The fusion image of T2-weighted imaging and DWIBS showed high signal intensity in the wall of the gallbladder. The high signal intensity with DWIBS indicated inflammation. (D) A thickened gallbladder wall was also observed in computed tomography images. Arrows indicate the gallbladder wall. All the figures were representative of the participants in the present study. DWIBS, diffusion-weighted whole body imaging with background body signal suppression.
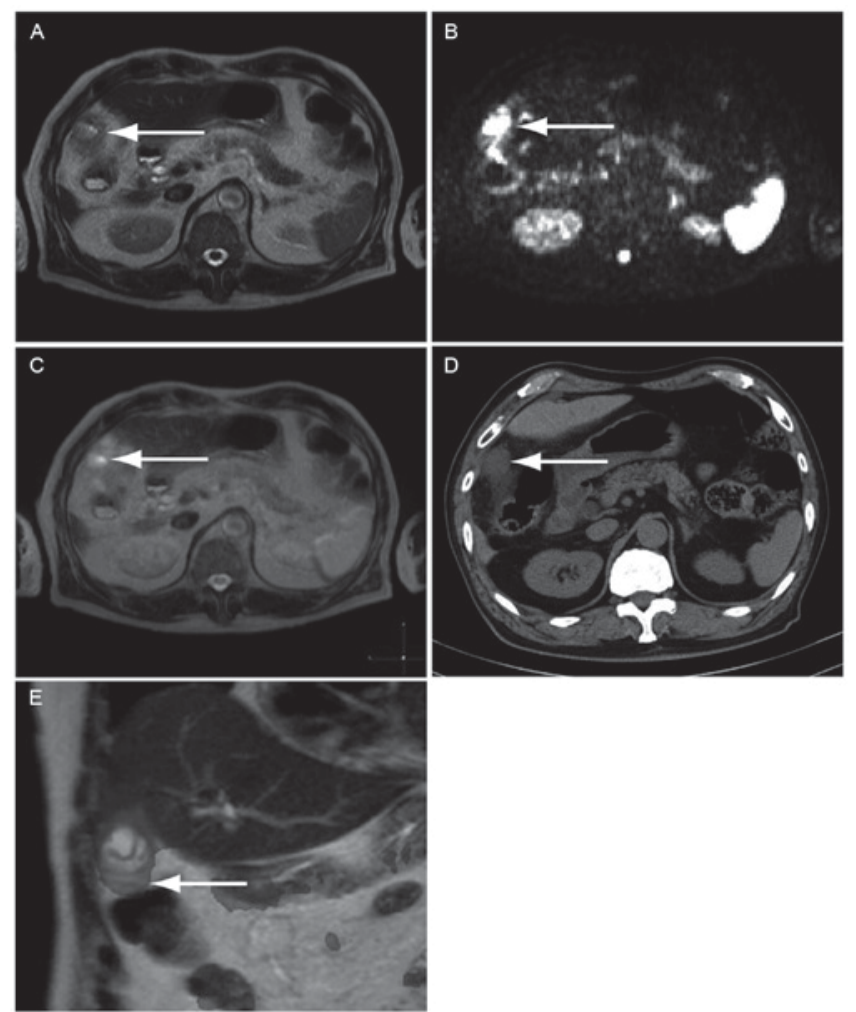

Figure 2. Pericholecystic inflammation. An 86-year-old man presented with upper abdominal pain. (A) T2-weighted imaging revealed a pericholecystic space. (B) DWIBS showed an area of high signal intensity in the pericholecystic space. (C) Anatomical analysis of the area of high signal intensity was conducted using DWIBS/T2. (D) Computed tomography images contained a low-density area in the pericholecystic space. (E) A cross section of DWIBS/T2 revealed pericholecystic inflammation. Arrows indicate the area of pericholecystic inflammation. All the figures were representative of the participants in the present study. DWIBS, diffusion-weighted whole body imaging with background body signal suppression; DWIBS/T2, DWIBS and $\mathrm{T} 2$ image fusion.
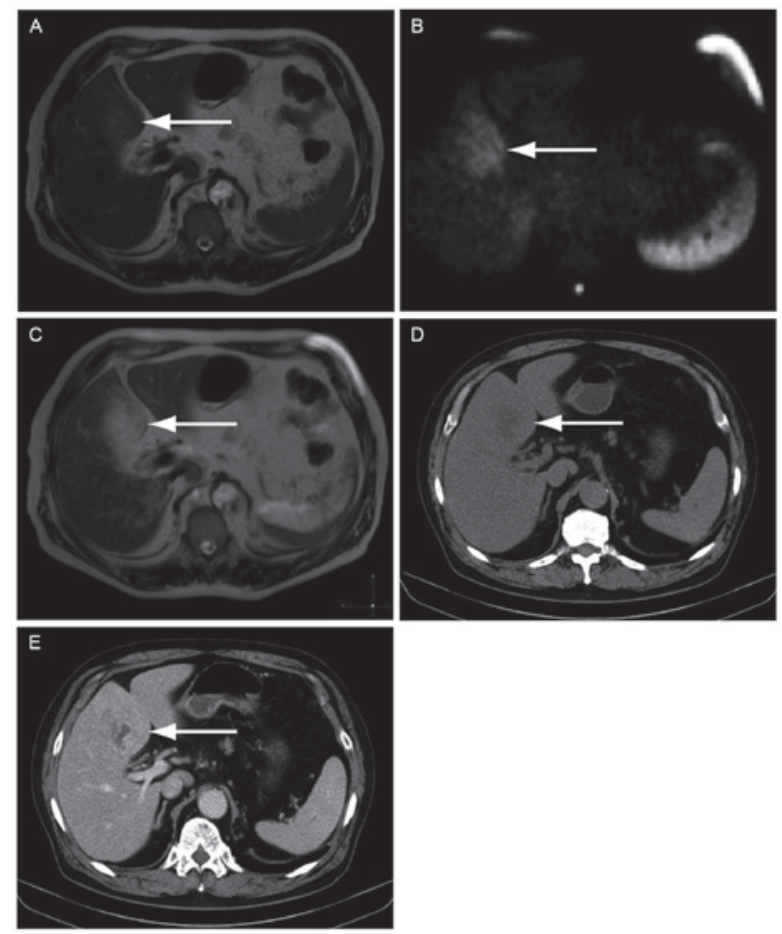

Figure 3. Liver inflammation due to acute cholecystitis. (A) A T2-weighed image of a 70-year-old man with acute cholecystitis found a weak high signal intensity in the liver. (B) High signal intensity was observed in the liver with DWIBS. (C) The fusion image of T2-weighted images and DWIBS clearly showed the high signal intensity in the liver. (D) Plain CT scanning identified an irregularly shaped, low-density area in the liver. (E) The low-density area became more obvious with contrast-enhanced CT. Arrows indicate the area of liver inflammation. All the figures were representative of the participants in the present study. DWIBS, diffusion-weighted whole body imaging with background body signal suppression; CT, computed tomography.

\section{Results}

The thickness of the gallbladder wall in DWIBS/T2 and CT images was compared. T2-weighted imaging revealed a thickened wall (Fig. 1A), and the signal intensity of the thickened wall was high with DWIBS (Fig. 1B). Fusion images of DWIBS and T2-weighted images showed high signal intensity of the gallbladder wall and allowed for anatomical analysis (Fig. 1C), which was not possible from the DWIBS image alone. The high signal intensity with DWIBS indicated inflammation. A thickened gallbladder wall was also observed in the CT images (Fig. 1D). These results suggest that gallbladder wall thickening is easily detectable using both DWIBS/T2 and CT imaging.

Pericholecystic inflammation is one of the complications of acute cholecystitis (9). T2-weighted imaging revealed a space around the gallbladder (Fig. 2A), which gave a high signal intensity with DWIBS (Fig. 2B). DWIBS/T2 showed high signal intensity for the gallbladder wall (Fig. 2C). The pericholecystic inflammation appeared as a low-density area in the CT images (Fig. 2D). The high signal intensity with DWIBS/T2 enabled easier detection than with CT, indicating that DWIBS/T2 shows pericholecystic inflammation more clearly than CT (Fig. 2E).

Liver abscess and liver inflammation are additional complications of acute cholecystitis (9). A weak high intensity signal was observed in the T2-weighted images for the liver (Fig. 3A). 
Table II. Patient characteristics.

\begin{tabular}{|c|c|c|c|c|c|c|c|c|}
\hline \multirow[b]{2}{*}{ Patient no. } & \multirow[b]{2}{*}{ Age, years } & \multirow[b]{2}{*}{ Sex } & \multicolumn{2}{|c|}{ Liver inflammation } & \multicolumn{2}{|c|}{$\begin{array}{l}\text { Pericholecystic } \\
\text { changes }\end{array}$} & \multicolumn{2}{|c|}{ Wall thickening } \\
\hline & & & DWIBS/T2 & $\mathrm{CT}$ & DWIBS/T2 & CT & DWIBS/T2 & $\mathrm{CT}$ \\
\hline 1 & 69 & $\mathrm{M}$ & $(-)$ & $(-)$ & $(-)$ & $(-)$ & $(+)$ & $(+)$ \\
\hline 2 & 70 & $\mathrm{M}$ & $(-)$ & $(-)$ & $(-)$ & $(-)$ & $(+)$ & $(+)$ \\
\hline 3 & 68 & $\mathrm{M}$ & $(-)$ & $(-)$ & $(-)$ & $(-)$ & $(+)$ & $(+)$ \\
\hline 4 & 69 & M & $(-)$ & $(-)$ & $(-)$ & $(-)$ & $(+)$ & $(+)$ \\
\hline 5 & 79 & $\mathrm{M}$ & $(+)$ & $(-)$ & $(+)$ & $(+)$ & $(+)$ & $(+)$ \\
\hline 6 & 65 & $\mathrm{M}$ & $(-)$ & $(-)$ & $(+)$ & $(+)$ & $(+)$ & $(+)$ \\
\hline 7 & 69 & $\mathrm{M}$ & $(-)$ & $(-)$ & $(+)$ & $(+)$ & $(+)$ & $(+)$ \\
\hline 8 & 70 & $\mathrm{~F}$ & $(-)$ & $(-)$ & $(+)$ & $(+)$ & $(+)$ & $(+)$ \\
\hline 9 & 76 & $\mathrm{~F}$ & $(+)^{\mathrm{a}}$ & $(+)^{\mathrm{a}}$ & $(-)$ & $(-)$ & $(+)$ & $(+)$ \\
\hline 10 & 70 & M & $(+)$ & $(-)$ & $(-)$ & $(-)$ & $(+)$ & $(+)$ \\
\hline 11 & 72 & $\mathrm{~F}$ & $(-)$ & $(-)$ & $(-)$ & $(-)$ & $(-)$ & $(-)^{\mathrm{b}}$ \\
\hline 12 & 88 & M & $(-)$ & $(-)$ & $(+)$ & $(+)$ & $(+)$ & $(+)$ \\
\hline 13 & 50 & $\mathrm{~F}$ & $(-)$ & $(-)$ & $(+)$ & $(+)$ & $(+)$ & $(+)$ \\
\hline 14 & 50 & $\mathrm{~F}$ & $(-)$ & $(-)$ & $(-)$ & $(-)$ & $(+)$ & $(+)$ \\
\hline
\end{tabular}

M, male; F, female; DWIBS/T2, diffusion-weighted whole body imaging with background body signal suppression/T2 image fusion; CT,

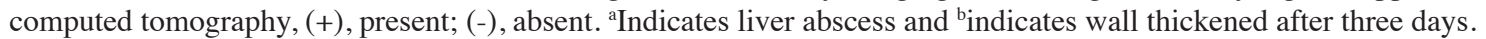

Table III. Laboratory test variables.

\begin{tabular}{|c|c|c|c|c|c|c|}
\hline \multirow[b]{2}{*}{ Parameter } & \multicolumn{3}{|c|}{ Liver inflammation } & \multicolumn{3}{|c|}{ Pericholecystic changes } \\
\hline & $(+), n=3$ & $(-), n=11$ & P-value & $(+), n=6$ & $(-), n=8$ & P-value \\
\hline WBC, $\mathrm{x} 10^{3} / \mathrm{ml}$ & $17.4 \pm 11.2$ & $9.8 \pm 4.3$ & 0.08 & $11.0 \pm 4.3$ & $12.0 \pm 8.3$ & 0.76 \\
\hline CRP, mg/dl & $15.5 \pm 5.6$ & $6.2 \pm 2.4$ & 0.09 & $8.4 \pm 10.6$ & $8.1 \pm 7.2$ & 0.95 \\
\hline ALP, IU/1 & $645 \pm 204$ & $462 \pm 106$ & 0.44 & $367 \pm 257$ & $602 \pm 389$ & 0.25 \\
\hline AST, IU/l & $63 \pm 20$ & $123 \pm 75$ & 0.69 & $36 \pm 25$ & $168 \pm 284$ & 0.28 \\
\hline ALT, IU/1 & $77 \pm 20$ & $85 \pm 183$ & 0.94 & $37 \pm 30$ & $119 \pm 210$ & 0.37 \\
\hline$\gamma$-GTP, IU/1 & $226 \pm 70$ & $232 \pm 273$ & 0.97 & $126 \pm 83$ & $320 \pm 313$ & 0.17 \\
\hline LDH, IU/1 & $214 \pm 40$ & $300 \pm 154$ & 0.37 & $228 \pm 62$ & $305 \pm 162$ & 0.40 \\
\hline $\mathrm{BUN}, \mathrm{mg} / \mathrm{dl}$ & $16 \pm 6$ & $17 \pm 2$ & 0.78 & $14 \pm 5$ & $17 \pm 6$ & 0.35 \\
\hline $\mathrm{Cre}, \mathrm{mg} / \mathrm{dl}$ & $0.75 \pm 0.25$ & $0.82 \pm 0.16$ & 0.59 & $0.82 \pm 0.14$ & $0.79 \pm 0.20$ & 0.78 \\
\hline
\end{tabular}

Data are presented as the mean \pm standard deviation. (+), detected; (-), not detected; WBC, white blood cell count; Hb, hemoglobin; CRP, C-reactive protein; ALP, alkaline phosphatase; AST, aspartate aminotransferase: ALT, alanine aminotransferase; $\gamma$-GTP gamma-glutamyl transpeptidase; $\mathrm{LDH}$, lactate dehydrogenase; BUN, blood urea nitrogen; Cre, creatinine.

An area with high signal intensity was observed for the liver with DWIBS (Fig. 3B). DWIBS/T2 images, however, clearly showed high signal intensity in the liver (Fig. 3C). The high intensity with DWIBS/T2 shown in Fig. 3C was less obvious than Figs. $1 \mathrm{C}$ and $2 \mathrm{C}$. The possible reason was that the intensity of the inflammation was less severe in that shown in Fig. 3C compared with Figs. 1C and 2C. A low-density area was observed in liver when plain CT was used (Fig. 3D), and contrast-enhanced CT made this low-density area more obvious (Fig. 3E).

Detectability of inflammation of the pericholecystic space and the liver were compared between DWIBS/T2 and
CT images (Table II). There was no obvious difference in detectability for either condition between DWIBS/T2 and CT.

The correlation between positive DWIBS/T2 results and laboratory test variables was also investigated (Table III). White blood count and CRP levels tended to be higher in patients whose DWIBS/T2 images indicated inflammation or liver abscesses; however, this difference was not statistically significant. These results suggested that acute cholecystitis is more severe in patients for whom there is a high signal density on the DWIBS/T2 image. DWIBS/T2 may therefore be more useful for the detection of severe acute cholecystitis. 


\section{Discussion}

MRI imaging typically reveals gallbladder wall thickening and pericholecystic fluid in patients with acute cholecystitis (23), and is reported to be superior to $\mathrm{CT}$ for the diagnosis of acute cholecystitis (24). In the present study, DWIBS/T2 was as successful as CT imaging in identifying wall thickening and pericholecystic inflammation. Furthermore, compared with CT, DWIBS/T2 showed positive results more clearly due to the strong contrast between the target and surrounding tissues.

Liver abscesses typically appear as an irregularly shaped, low-density area, with slight signal enhancement in surrounding tissues (25). One patient in the present study had a liver abscess, and this was easily diagnosed via DWIBS/T2 or CT scanning. For this patient, a small liver abscess was observed in a contrast-enhanced CT image. DWIBS/T2 revealed an area of high signal intensity around the abscess. These results indicate that, in this case, DWIBS/T2 was able to reveal an area of inflammation in the liver that was developing into a liver abscess. The high signal intensity area observed with DWIBS/T2 appeared as a slight enhancement in the CT image, and was easy to detect. However, DWIBS/T2 indicated the area more clearly as the high signal intensity was strongly contrasted against the surrounding tissues. These results suggested that DWIBS/T2 enables easier evaluation of liver abscesses and the surrounding inflammation compared with CT.

One limitation of the present study was the small number of patients. Furthermore, the other complications that typically occur with cholecystitis, such as gallbladder perforation, emphysema, and gangrene, were not investigated. In future studies, a greater number of patients should be included to evaluate a broader set of complications.

In conclusion, the results of the present study suggest that DWIBS/T2 has the same sensitivity as CT for the diagnosis of complicated acute cholecystitis. However, the strong contrast shown by DWIBS/T2 allows for easier evaluation of acute cholecystitis than CT scanning. These findings may be beneficial in a clinical setting as they allow doctors to select the most effective diagnostic imaging technique for patients with suspected cholecystitis.

\section{References}

1. Knab LM, Boller AM and Mahvi DM: Cholecystitis. Surg Clin North Am 94: 455-470, 2014.

2. Pinto A, Reginelli A, Cagini L, Coppolino F, Stabile Ianora AA, Bracale R, Giganti M and Romano L: Accuracy of ultrasonography in the diagnosis of acute calculous cholecystitis: Review of the literature. Crit Ultrasound J 5 (Suppl 1): S11, 2013.

3. Singal R, Mittal A, Gupta S, Singh B and Jain P: Management of gall bladder perforation evaluation on ultrasonography: Report of six rare cases with review of literature. J Med Life 4: 364-371, 2011.

4. Tang S, Wang Y and Wang Y: Contrast-enhanced ultrasonography to diagnose gallbladder perforation. Am J Emerg Med 31: 1240-1243, 2013.

5. Koti RS, Davidson CJ and Davidson BR: Surgical management of acute cholecystitis. Langenbecks Arch Surg 400: 403-419, 2015.

6. Yokoe M, Takada T, Strasberg SM, Solomkin JS, Mayumi T, Gomi H, Pitt HA, Gouma DJ, Garden OJ, Büchler MW, et al: New diagnostic criteria and severity assessment of acute cholecystitis in revised Tokyo Guidelines. J Hepatobiliary Pancreat Sci 19: 578-585, 2012.
7. Myrianthefs P, Evodia E, Vlachou I, Petrocheilou G, Gavala A, Pappa M, Baltopoulos G and Karakitsos D: Is routine ultrasound examination of the gallbladder justified in critical care patients? Crit Care Res Pract 2012: 565617, 2012.

8. Teefey SA, Dahiya N, Middleton WD, Bajaj S, Dahiya N, Ylagan L and Hildebolt CF: Acute cholecystitis: Do sonographic findings and WBC count predict gangrenous changes? AJR Am J Roentgenol 200: 363-369, 2013.

9. Shakespear JS, Shaaban AM and Rezvani M: CT findings of acute cholecystitis and its complications. AJR Am J Roentgenol 194: 1523-1529, 2010.

10. Bonekamp S, Corona-Villalobos CP and Kamel IR: Oncologic applications of diffusion-weighted MRI in the body. J Magn Reson Imaging 35: 257-279, 2012.

11. Sehy JV, Ackerman JJ and Neil JJ: Apparent diffusion of water, ions, and small molecules in the Xenopus oocyte is consistent with Brownian displacement. Magn Reson Med 8: 42-51, 2002.

12. Koike N, Cho A, Nasu K, Seto K, Nagaya S, Ohshima Y and Ohkohchi N: Role of diffusion-weighted magnetic resonance imaging in the differential diagnosis of focal hepatic lesions. World J Gastroenterol 15: 5805-5812, 2009.

13. Takahara T, Imai Y, Yamashita T, Yasuda S, Nasu S and Van Cauteren M: Diffusion weighted whole body imaging with background body signal suppression (DWIBS): Technical improvement using free breathing, STIR and high resolution 3D display. Radiat Med 22: 275-282, 2004.

14. Kwee TC, Takahara T, Ochiai R, Nievelstein RA and Luijten PR: Diffusion-weighted whole-body imaging with background body signal suppression (DWIBS): Features and potential applications in oncology. Eur Radiol 18: 1937-1952, 2008.

15. Ohno Y, Koyama H, Onishi Y, Takenaka D, Nogami M, Yoshikawa T, Matsumoto S, Kotani Y and Sugimura K: Non-small cell lung cancer: Whole-body MR examination for M-stage assessment-utility for whole-body diffusion-weighted imaging compared with integrated FDG PET/CT. Radiology 248: 643-654, 2008.

16. Fischer MA, Nanz D, Hany T, Reiner CS, Stolzmann P, Donati OF, Breitenstein S, Schneider P, Weishaupt D, von Schulthess GK and Scheffel H: Diagnostic accuracy of whole-body MRI/DWI image fusion for detection of malignant tumours: A comparison with PET/CT. Eur Radiol 21: 246-255, 2011.

17. Sommer G, Wiese M, Winter L, Lenz C, Klarhöfer M, Forrer F, Lardinois D and Bremerich J: Preoperative staging of non-small-cell lung cancer: Comparison of whole-body diffusion-weighted magnetic resonance imaging and 18F-fluorodeoxyglucose-positron emission tomography/computed tomography. Eur Radiol 22: 2859-2867, 2012.

18. Nechifor-Boilă IA, Bancu S, Buruian M, Charlot M, Decaussin-Petrucci M, Krauth JS, Nechifor-Boilă AC and Borda A: Diffusion weighted imaging with background body signal suppression/T2 image fusion in magnetic resonance mammography for breast cancer diagnosis. Chirurgia (Bucur) 108: 199-205, 2013

19. Tomizawa M, Shinozaki F, Motoyoshi Y, Sugiyama T, Yamamoto $\mathrm{S}$ and Ishige N: Diffusion-weighted whole body imaging with background body signal suppression/T2 image fusion is negative for patients with intraductal papillary mucinous neoplasm. Hepatogastroenterology 62: 463-465, 2015.

20. Hirota M, Takada T, Kawarada Y, Nimura Y, Miura F, Hirata K, Mayumi T, Yoshida M, Strasberg S, Pitt H, et al: Diagnostic criteria and severity assessment of acute cholecystitis: Tokyo guidelines. J Hepatobiliary Pancreat Surg 14: 78-82, 2007.

21. Ralls PW, Halls J, Lapin SA, Quinn MF, Morris UL and Boswell W: Prospective evaluation of the sonographic Murphy sign in suspected acute cholecystitis. J Clin Ultrasound 10: 113-115, 1982.

22. Wang Y, Miller FH, Chen ZE, Merrick L, Mortele KJ, Hoff FL, Hammond NA, Yaghmai V and Nikolaidis P: Diffusion-weighted MR imaging of solid and cystic lesions of the pancreas. Radiographics 31: E47-E64, 2011.

23. Tonolini M, Ravelli A, Villa C and Bianco R: Urgent MRI with MR cholangiopancreatography (MRCP) of acute cholecystitis and related complications: Diagnostic role and spectrum of imaging findings. Emerg Radiol 19: 341-348, 2012.

24. Kaura SH, Haghighi M, Matza BW, Hajdu CH and Rosenkrantz $\mathrm{AB}$ : Comparison of $\mathrm{CT}$ and MRI findings in the differentiation of acute from chronic cholecystitis. Clin Imaging 37: 687-691, 2013.

25. Feier D, Socaciu M, Anton O, Al Hajjar N and Badea R: The combined role of intravenous contrast enhanced ultrasound (CEUS) and computed tomography (CT) in liver abscess diagnosis. Chirurgia (Bucur) 107: 343-351, 2012. 